\title{
Main Kafa, 2013, L'habitat informel en Syrie, le cas de
}

\section{Damas}

Thèse de géographie, Nancy, Université de Lorraine, école doctorale "Fernand Braudel", LOTERR Centre de recherche en géographie, $296 \mathrm{p}$.

Jean-Marie Simon

\section{(2) OpenEdition}

\section{Journals}

Electronic version

URL: http://journals.openedition.org/rge/5118

DOI: 10.4000/rge.5118

ISSN: $2108-6478$

\section{Publisher}

Association des géographes de l'Est

Printed version

Date of publication: 30 December 2013

ISSN: 0035-3213

\section{Electronic reference}

Jean-Marie Simon, « Main Kafa, 2013, L'habitat informel en Syrie, le cas de Damas », Revue Géographique de l'Est [Online], vol. 53 / 3-4 | 2013, Online since 06 July 2014, connection on 23 September 2020. URL : http://journals.openedition.org/rge/5118; DOI : https://doi.org/10.4000/rge.5118

This text was automatically generated on 23 September 2020 .

Tous droits réservés 


\title{
Main Kafa, 2013, L'habitat informel en Syrie, le cas de Damas
}

Thèse de géographie, Nancy, Université de Lorraine, école doctorale "Fernand Braudel", LOTERR Centre de recherche en géographie, $296 \mathrm{p}$.

\author{
Jean-Marie Simon
}

\section{REFERENCES}

Main Kafa, 2013, L'habitat informel en Syrie, le cas de Damas, Thèse de géographie, Nancy, Université de Lorraine, école doctorale "Fernand Braudel", LOTERR Centre de recherche en géographie, $296 \mathrm{p}$.

La thèse de doctorat en géographie de Monsieur Main Kafa, menée sous la direction du Professeur André Humbert de l'université de Lorraine, a été soutenue à Nancy le 16 décembre 2013. Le jury était composé du professeur Marcel Bazin (Univeristé de Reims Champagne-Ardennes), du doyen Hassan Benhalima (Université d'Agadir, Maroc), tous deux rapporteurs, du professeur André Humbert (directeur) et du professeur JeanPierre Husson (Université de Lorraine, président de séance). L'auteur fournit au jury un volume publié recto-verso de 330 pages, illustré et accompagné d'une bibliographie trilingue. Main Kafa, diplômé de l'école d'architecture d'Alep, décrit et analyse les quartiers construits en dehors des règles d'urbanisme, qui constituent aujourd'hui 40\% des logements de la capitale syrienne. La thèse, engagée il y a quelques années, aboutit alors que la guerre civile touche plusieurs de ces quartiers, sans qu'il soit encore possible de dire si les reconstructions à venir modifieront les pratiques identifiées dans la thèse. En dépit d'une sensibilité parfois perceptible pour l'avenir de son pays, l'auteur demeure rigoureux et objectif dans ses approches urbaines. Il propose de regarder ces quartiers comme une réponse pragmatique aux besoins de logements de la population d'une capitale adossée à la montagne (le Qassioum) et peinant à protéger son oasis (la Ghouta). 
2 La thèse s'organise en trois parties, présentation générale de la ville, description de ces quartiers «d'habitat informel», et perspectives d'évolution. L'approche démographique, bien développée dans la première partie, montre que la croissance est due à l'exode rural propre aux pays en développement, mais aussi aux arrivées successives de réfugiés, Syriens du Golan, Palestiniens, Kurdes puis Irakiens. Les besoins en logements sont donc extrêmement importants et donnent naissance à des quartiers qui maintiennent des liens communautaires.

3 La deuxième partie, sans doute la plus originale, apporte des éléments de connaissance concrète sur la réalité de ces quartiers. En effet on ne dispose guère d'informations sur ces pratiques «illégales » et les enquêtes sont confrontées à des personnes forcément prudentes dans leurs réponses. Main Kafa a pratiqué ses quartiers à la fois à titre personnel (il y a habité) et professionnel car, architecte, il a travaillé sept années dans l'administration en charge du logement à Damas. Il a, grâce à ses contacts, conduit des enquêtes et visites de terrain qui sont une source précieuse d'informations. L'auteur a ainsi construit un échantillon de 260 logements répartis dans 159 bâtiments. Onze quartiers font l'objet de descriptions fines : situation par rapport à la ville « officielle ", états des voiries, des réseaux et des équipements. Le travail se poursuit par l'examen des procédures de lotissements « clandestins » et les raisons pour lesquelles l'étalement urbain se fait sur l'oasis de la Ghouta et non sur les plateaux prévus pour l'urbanisation officielle. Au-delà des procédures d'acquisition, l'enquête décrit avec beaucoup de soin l'organisation intérieure des habitations, les étapes et les techniques de construction. L'ensemble de ces dispositions explique que le coût de l'opération s'établit au vingtième d'une construction respectant les règlements d'urbanisme. Mais il ne s'agit pas pour autant des "bidonvilles" que connaissent certaines villes des pays en voie de développement. Cet habitat est en effet destiné à durer, voire à se développer progressivement en offrant des conditions de vie acceptables. Cette situation paraitrait une réponse pertinente à la question du logement si les espaces et services urbains n'étaient pas réduits au strict minimum. Un des objectifs des habitants et des communautés de ces quartiers est donc d'obtenir une amélioration des services urbains et une régularisation de leur situation.

$4 \mathrm{M}$. Kafa consacre la troisième partie de sa thèse à ces questions de réhabilitation et la régularisation de ces quartiers. Ces pratiques ne sont pas forcément à considérer comme anormales, en effet dans l'ensemble des villes syriennes plus de $50 \%$ des logements se construisent ainsi. Les régularisations, pratiques déjà anciennes, sont effectuées quartier par quartier et certains secteurs sont même devenus des quartiers aisés comme à Berzeh. Il ne serait pas illogique de penser que ces pratiques vernaculaires sont une étape courante de la croissance urbaine dans ce pays. Mais ces régularisations, mêmes si elles relèvent d'une certaine tradition urbaine renforcent les pratiques de clientélisme et mettent en situation de dépendance forte les habitants.

5 La thèse qui se termine par une belle bibliographie de plus de trois cents références, incite à une large réflexion sur les questions d'urbanisme. Un comparatif critique des formes urbaines entre la ville ancienne, celle des projets de Michel Ecochard (1968) et celles de ces "quartiers informels» devrait apporter des éléments de réponse pour l'avenir urbain de la capitale syrienne. À un niveau plus général, un travail théorique sur cette notion d'habitat informel devra interroger les pratiques de l'urbanisme dans leur ensemble. La première question est relative à la capacité d'une population à ignorer, voire contourner, les règles d'urbanisme pour répondre à des besoins 
fondamentaux; il s'agit partiellement d'un problème d'ordre politique, mais les professionnels doivent aussi rechercher des solutions urbaines adaptées aux capacités financières et culturelles des populations. L'autre question est relative à la pratique du clientélisme, vers des individus ou des groupes; lors de l'élaboration et la mise en œuvre des plans d'urbanisme, une partie de la réponse est sans doute dans la concertation et la transparence mais cela demande encore à être démontré.

\section{AUTHORS}

JEAN-MARIE SIMON

Doctorant en géographie à l'Université de Lorraine 